\title{
Dilute suspensions in annular shear flow under gravity: simulation and ex- periment
}

\author{
Kevin Schröer ${ }^{1, \star}$, Patrick Kurzeja ${ }^{1}$, Stephan Schulz ${ }^{1}$, Philipp Brockmann², Jeanette Hussong ${ }^{2}$, Peter Janas ${ }^{3}$, Ire- \\ naeus Wlokas ${ }^{3}$, Andreas Kempf ${ }^{3}$, and Dietrich E. Wolf ${ }^{1}$ \\ ${ }^{1}$ Faculty of Physics and CENIDE, University of Duisburg-Essen, Duisburg, Germany \\ ${ }^{2}$ Institute for Thermo and Fluid Dynamics - Hydraulic Fluid Machinery, Ruhr-University Bochum, Germany \\ ${ }^{3}$ Institute for Combustion and Gas Dynamics - Fluid Dynamics and CENIDE, University of Duisburg-Essen, Duisburg, Germany
}

\begin{abstract}
A dilute suspension in annular shear flow under gravity was simulated using multi-particle collision dynamics (MPC) and compared to experimental data. The focus of the analysis is the local particle velocity and density distribution under the influence of the rotational and gravitational forces. The results are further supported by a deterministic approximation of a single-particle trajectory and OpenFOAM CFD estimations of the overcritical frequency range. Good qualitative agreement is observed for single-particle trajectories between the statistical mean of MPC simulations and the deterministic approximation. Wall contact and detachment however occur earlier in the MPC simulation, which can be explained by the inherent thermal noise of the method. The multi-particle system is investigated at the point of highest particle accumulation that is found at $2 / 3$ of the particle revolution, starting from the top of the annular gap. The combination of shear flow and a slowly rotating volumetric force leads to strong local accumulation in this section that increases the particle volume fraction from overall $0.7 \%$ to $4.7 \%$ at the outer boundary. MPC simulations and experimental observations agree well in terms of particle distribution and a close to linear velocity profile in radial direction.
\end{abstract}

\section{Introduction \& Goals}

Particle-laden flows in narrow annular gaps of hydraulic fluid machinery may alter the systems' performance even at moderate seeding densities. However, the detailed particle behavior and underlying mechanisms such as local particle accumulation or particle segregation effects in TaylorCouette flows of narrow gaps are still little understood up to date $[1,2]$.

Various numerical and experimental approaches have been developed to analyze complex suspensions, including combined discrete element and finite volume methods [3] or imaging techniques [4]. A young simulation technique emerged from the so-called multi-particle collision dynamics method (MPC) that mimics the fluid by coarsegrained particles and a simplified collision model [5]. The MPC technique fully incorporates hydrodynamic interactions as well as thermal fluctuations and benefits from a highly parallelized numerical implementation [6]. The present study aims to develop numerical and experimental complementary methods to study the particle dynamics of a dilute, sheared, rotating suspension under the influence of gravity. It focuses on the local particle velocity and density variations while verifying the MPC method as a predictive, stochastic tool by means of an especially adapted A-PTV method. A finite volume CFD simulation is additionally used to determine the threshold for over-

\footnotetext{
^e-mail: kevin.schroeer@uni-due.de
}

critical laminar flow. After introducing the methodology, we investigate a single-particle simulation and compare it with a deterministic approximation. Afterwards, we compare a multi-particle simulation with experimental results at the position of highest local particle density. Special attention is given to the capabilities and limitations of the MPC method.

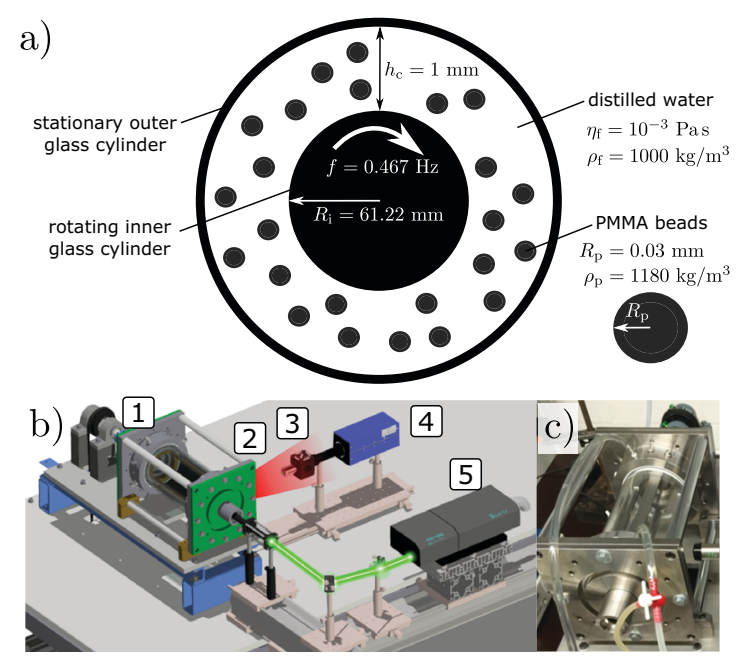

Figure 1. a) Sketch of the system investigated by experiment and simulations (not to scale); b) components of experimental setup: 1 Taylor-Couette cell, 2 microscope objective, 3 microscope tubus, 4 CCD camera, 5 Nd:YAG laser; c) close up of the Taylor-Couette cell. 


\section{Methods}

\subsection{System of investigation and dimensionless characterization}

Our setup consists of two concentrically and horizontally aligned glass cylinders with $1 \mathrm{~mm}$ spacing that will be called the Taylor-Couette cell, see Fig. 1. Its inner cylinder rotates with $f=0.467 \mathrm{~Hz}\left(\sim v_{0}=0.18 \mathrm{~m} / \mathrm{s}\right.$ at its outer edge), while the outer cylinder is stationary. The annular gap between both cylinders is filled with distilled water and monodisperse PMMA beads of $60 \mu \mathrm{m}$ diameter. The ratio of channel height $\left(h_{\mathrm{c}}=1 \mathrm{~mm}\right)$ to the radius of the inner cylinder $\left(R_{\mathrm{i}}=61.22 \mathrm{~mm}\right)$ is 0.016 and thus minimizes the influence of curvature when focusing on a local section.

The two dimensionless key parameters of the problem at hand are the Froude number Fr $=v_{0}^{2} /\left(R_{\mathrm{i}} g\right)=0.055$, balancing the amplitudes of centrifugal and gravitational forces, and the dimensionless frequency $f^{*}=f h^{2} \rho_{\mathrm{f}} / \eta_{\mathrm{f}}=$ $4.5 \cdot 10^{-7}$, i.e. the ratio of viscous relaxation time to the rotation period (with $g=9.81 \mathrm{~m} / \mathrm{s}^{2}$, fluid density $\rho_{\mathrm{f}}=$ $1000 \mathrm{~kg} / \mathrm{m}^{3}$ and dynamic viscosity $\eta_{\mathrm{f}}=0.001 \mathrm{~Pa} \mathrm{~s}$ ). These parameters place the presented setup between pure vertically aligned sedimentation $(\mathrm{Fr} \rightarrow 0)$ and radially oriented centrifugation $(\mathrm{Fr} \rightarrow \infty)$. The particles are slightly denser compared to the fluid $\left(\rho_{\mathrm{s}} / \rho_{\mathrm{f}}=1.18\right)$. Very low characteristic Mach and channel Reynolds numbers, $\mathrm{Ma}=$ $v_{0} / c_{\text {sound }}=1.25 \cdot 10^{-4}$ and $\operatorname{Re}_{\mathrm{ch}}=v_{0} h_{\mathrm{c}} \rho_{\mathrm{f}} / \eta_{\mathrm{f}}=190$, support the assumption of incompressible, laminar flow. The particle Reynolds number based on the shearing rate is $\operatorname{Re}_{\mathrm{p}}=\left(v_{0} / h_{\mathrm{c}}\right) R_{\mathrm{p}}^{2} \rho_{\mathrm{f}} / \eta_{\mathrm{f}}=0.162$. Thermal fluctuations are furthermore negligible owing to the large particle Peclet number of $\mathrm{Pe}=\left(3 \pi R_{\mathrm{p}}^{3} \eta_{\mathrm{f}} v_{0}\right) /\left(2 h_{\mathrm{c}} k_{\mathrm{B}} T\right)=2.26 \cdot 10^{7}$ at room temperature.

\subsection{MPC Simulation}

The MPC method mimics the fluid in a stochastically coarse-grained manner by mesoscopic fluid point particles [5]. These fluid particles alternately undergo a streaming and a collision step. During the streaming step, the fluid particles move independently, keeping their velocity. During the collision step, particles are sorted into a grid of collision cells, and their velocities relative to the cell's mean velocity are rotated about a rotation axis randomly generated for each cell and time step. This collision rule, known as stochastic rotation dynamics (SRD), ensures local momentum and energy conservation. In the current numerical setting, the collision-cell width is chosen to be $0.01 h_{\mathrm{c}}$ with 20 fluid particles per cell. The rotation angle of $100^{\circ}$ and the mean free path of 0.32 lead to $\Delta t_{\mathrm{MPC}}=11.24 \mathrm{~ms}$.

The suspended solid particles are integrated by the Velocity Verlet algorithm imposing $\Delta t_{\mathrm{MD}}=0.1 \Delta t_{\mathrm{MPC}}$ and particle-particle interaction via a hard-sphere potential. Fluid-particle coupling is achieved by a bounce-back rule during the streaming step and the use of virtual particles during the collision step [7].

Instead of the full geometry, a cubic box of side length $h_{\mathrm{c}}$ is simulated, neglecting curvature of the boundaries.
The simulation box is interpreted as a reference frame that is moving along the cylinder gap with mean fluid velocity $v_{0} / 2$. The box boundaries are shearing rigid walls in radial direction and periodic boundaries in all other directions. In addition to the gravitational force, Coriolis and centrifugal forces are applied to the suspended colloids. Following the reference frame, the gravitational force is rotated accordingly in order to recover its varying influence at different angular positions along the cylinder. In the case of a single particle, rotation is adjusted by the global particle position. Because incompressibility in the MPC fluid is not assured, applying the above mentioned forces to the fluid particles would lead to non-physical artifacts. Instead, effective buoyancy is taken into account according to the colloid-fluid density mismatch.

\subsection{Experiment}

Centrifugal and gravity-driven particle dynamics were studied in an optically accessible Taylor-Couette setup by means of shadowgraphy imaging (see Fig. 1 b)). A frequency-doubled dual cavity Nd:YAG laser (New Wave Solo III-15) with $532 \mathrm{~nm}$ wavelength and $8 \mathrm{~ns}$ pulse length was used for volume illumination. The laser beam was guided into the Taylor-Couette setup to excite a red fluorescent color-coated plate. In this way, incoherent fluorescence light is emitted passing through inner and outer glass cylinders in positive radial direction to create a homogeneous, speckle-free background illumination. Images were taken with a 12 bit dual frame CCD-cameras (Imager Intense, LaVision) with 1376 x 1040 image size. Using a microscope objective of 5x magnification and 12 $\mu \mathrm{m}$ focal depth (CFI60 TU Plan (MUE12050), Nikon), an image resolution of $1.26 \mu \mathrm{m} /$ pixel is reached, yielding a field of view of $1.76 \mathrm{~mm} \times 1.32 \mathrm{~mm}$. A suspension of PMMA particles (Microbeads AS; final diameter as provided by the manufacturer of $62.8 \mu \mathrm{m} \pm 1.2 \%$ standard deviation) was injected in the inlet of the system briefly before the experiment was initiated. Fifty double-frame images were taken every $5 \mu \mathrm{m}$ along the gap width. A 3D-PTV procedure was developed similar to Cierpka et al. [8] to achieve an out-of plane particle position reconstruction. Radial particle positions could be determined with an overall standard deviation of $34 \mu \mathrm{m}$ accuracy. The reference position at the outer cylinder was determined by reducing invalid detections outside the channel to less than $4 \%$ of all particles.

\subsection{Finite Volume Simulation}

As a second numerical approach, the 3D-CFD software OpenFOAM was used to estimate the transition range towards Taylor-Couette-instabilities [3]. The convective fluxes are discretized by a total variation diminishing scheme (TVD) with the Sweby limiter. A larger simulation box of $5 \mathrm{~mm} \times 5 \mathrm{~mm} \times 1 \mathrm{~mm}$ was chosen to account for the system's curvature and thus implicitly consider centrifugal and Coriolis forces. No-slip boundary conditions are applied to the cylinder walls and periodic boundaries are used in axial and circumferential direction. 


\subsection{Deterministic approximation of the single-particle trajectory}

The steady-state trajectory of a single particle was theoretically approximated by Newton's second law and solved numerically using MATLAB's ode solver ode15s. The governing equations account for inertia, Stokes drag, gravitation, the Saffman lift force [9] and the virtual-mass force [10]. A stationary flow field, elastic slip motion at contact with the outer wall and negligible angular motion of the particle were assumed. Coriolis and Basset forces were neglected, because of small radial and relative velocities between particle and fluid.

\section{Results and Discussion}

\subsection{Methodological peculiarities and limitations}

We consider it helpful to discuss the major peculiarities and limitations of each method before comparing and evaluating the specific results. This clarification helps to justify the assumptions made and to highlight potentially deviating conditions beforehand.

Thermal noise incorporated in the MPC method reduces the Peclet number from $\mathrm{Pe}=2.26 \cdot 10^{7}$ in the experiment to 28 in the simulation. In the case of multiple particles, all particles are exposed to the same orientation of the gravity force that is determined by the location of the reference frame. This assumption is motivated by small curvature effects within the simulation box. Nevertheless, particles on an inner radial trajectory rotate faster and should experience a shorter force oscillation and vice versa. Using the mean fluid velocity for the reference frame is therefore assumed as a compromise.

The formation of a distinguished particle band perpendicular to the cylinder axis was observed in the experiment. This is a well-known phenomenon in particle-laden drum flows exceeding a particle-density threshold for intermediate frequencies [11]. In the present case, the band formation is $5 \mathrm{~mm}$ to $10 \mathrm{~mm}$ in thickness, depending on the circumferential position, and thus qualified as a homogeneous particle distribution on the length scale of $1 \mathrm{~mm}$ to be compatible with the assumptions of the numerical approaches. The measurements were hence performed in the centerline of the band to ensure the most periodic conditions possible.

Taylor-Couette-instabilities were prevented by keeping the channel Reynolds number $\mathrm{Re}_{\mathrm{ch}}=190$ below the critical value of $\operatorname{Re}_{\text {ch,crit }} \approx 350$, which was determined by a linear stability analysis. Accordingly, no Taylor vortices are observed for the operation point of the experiment. This was verified by the finite volume method via a parameter test that identified a transition at $\operatorname{Re}_{c h, c r i t} \geq 3800$.

\subsection{Single particle}

The deterministic single-particle approximation predicts a long-term trajectory, along which single particles will move independently of their starting position as illustrated in Fig. 2. The MPC simulation resembles this characteristic trajectory, distinguishing a convection-dominated drift period from a contact period at the lower left wall. Averaged over six revolutions, the simulation predicts closest approach to the wall approximately $25^{\circ}$ earlier. The individual retention of minimum particle-wall distance also fluctuates and is generally smaller in the MPC simulation. The comparably strong delay observed in the deterministic approximation may result from the simplifying assumptions yielding an underestimation of the total lift force [12]. A final quantitative evaluation exceeds the scope of this work, and the necessity of a more precise modeling of close contact will be discussed below against the background of multiple particles.

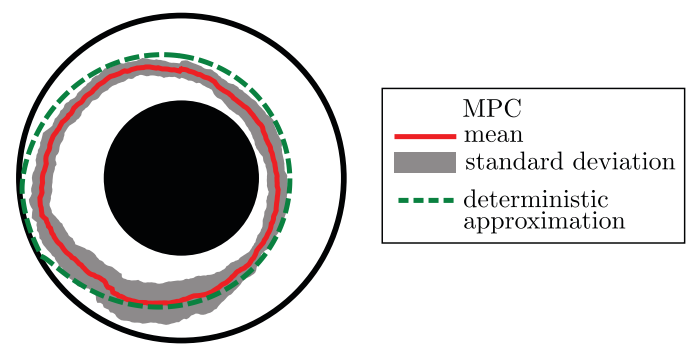

Figure 2. Comparison between steady-state deterministic approximation and MPC simulation of the trajectory of a single particle (not to scale, channel height is magnified).

\subsection{Multiple particles}

The study of multiple particles is focused on a section at $240^{\circ}$ clockwise revolution, where the single-particle results predict strongest particle-wall interaction and therefore the highest particle accumulation, see Fig. 3 a). The corresponding particle distribution over the annular gap derived from experiments and simulations are in good agreement, as can be seen in Fig. 3 b). The simulations were performed with 63 particles per $\mathrm{mm}^{3}$ corresponding to a mean particle volume fraction of $0.7 \%$ in this section as found in the experiment. However, most particles are located in an interval of approximately $15 \%$ gap-width centered around the maximum density at 1.5 particle diameters (i.e. $90 \mu \mathrm{m}$ ) outer wall distance, leading to a local volume fraction of $4.7 \%$. In this region, the slope of the almost linear particle velocity profile is slightly reduced, see the left $180 \mu \mathrm{m}$ interval in Fig. $3 \mathrm{c}$ ). This behavior can be qualitatively understood by particle-induced changes in viscosity, estimated by the Krieger model [13] to reach $13 \%$ deviation in this region of highest accumulation compared to only $2 \%$ for the section's mean volume fraction.

Particles do not adhere to the resting wall collectively. Their behaviour rather resembles the one of a single particle as observed from the MPC simulation. We assume that a wall-lift force prevents the particles from getting into direct contact with the wall [12]. Also direct contact or short-range hydrodynamic interaction between particles can enhance repulsion in the particle cloud.

We suggest that the accuracy of the present MPC simulation mainly depends on particle-fluid and particleparticle momentum exchange, because of the rare particlewall contacts. This is supported by the similarity between 
the stochastic MPC approach and the experimentally observed attributes of the particle and velocity distributions. Possible sources for quantitative deviations are the finite Pe number as well as periodic boundary conditions. Slight layering is also observed in the simulation, see the local peaks approximately one particle diameter apart in Fig. 3. This can be explained by the constant rotation period assumed for the numerically implemented reference frame that allows for a development of particle layers before break-up. Future studies extending the periodically confined simulation box will allow for a more accurate implementation of the gravity force acting individually on each particle such that the time-dependent formation can be predicted more precisely.

a)
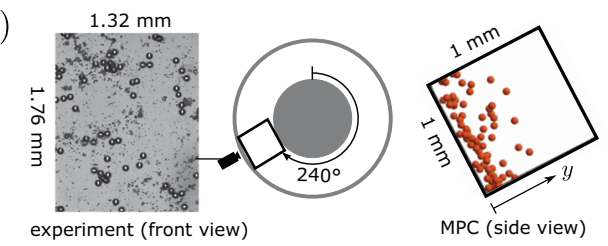

b)
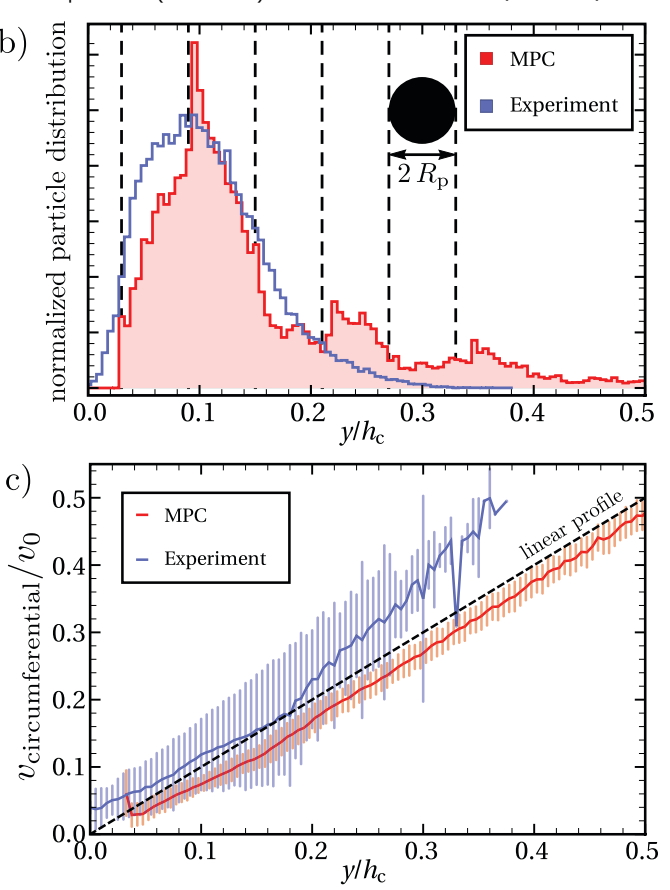

Figure 3. a) Position of the evaluation region at $240^{\circ}$ for the multi-particle study with snapshots of experimental and MPC data; b) density profile of the particle distribution per $5 \mu \mathrm{m}$ interval for experiment and MPC simulation normalized by total particle number and plotted over normalized radial distance $y$ from the outer cylinder; c) velocity profile for experiment and MPC simulation averaged over $5 \mu \mathrm{m}$-intervals with standard deviation as vertical lines plotted over normalized radial distance $y$ from the outer cylinder.

\section{Conclusion}

We studied dilute suspensions in annular shear flow numerically and experimentally with a ratio of radial centrifugal forces to vertical gravity forces of 0.055 . The single-particle trajectory predicted by the MPC simulation shows good agreement with the deterministic approximation during the distinct periods of free drift and wall contact. The closest approach to the wall and the subsequent release appeared earlier in the MPC simulation. This can be explained by thermal fluctuations that are inherent to the MPC approach and better reflect stochastic perturbations of the steady-state trajectory. The multi-particle system accordingly shows rare particle-wall contacts and a peak of the particle distribution about 1.5 diameters away from the outer wall, leading to a locally increased volume fraction $(0.7 \% \rightarrow 4.7 \%)$ and viscosity $(2 \% \rightarrow 13 \%)$. These characteristics are also well predicted by the MPC simulations; notwithstanding the algorithmic restrictions in terms of Peclet number, Mach number and periodic boundaries. Future studies will focus on the improvement of the MPC simulation in terms of larger Peclet numbers and a gravitational stimulation that acts individually on the timedependent position of each particle.

\section{Acknowledgements}

Funding by the Mercator Research Center Ruhr (project 2015-0021) is gratefully acknowledged. We thank Godehard Sutmann at Jülich Supercomputing Centre for providing fruitful discussions and the basic algorithm of the $\mathrm{MP}^{2} \mathrm{C}$ implementation. Patrick Kurzeja gratefully acknowledges financial support by DFG grant KU 3351/2-1.

\section{References}

[1] J. Lee, A.J. Ladd, Journal of Fluid Mechanics 577, 183 (2007)

[2] G. D'Avino, P. Maffettone, Journal of NonNewtonian Fluid Mechanics 215, 80 (2015)

[3] C. Kloss, C. Goniva, A. Hager, S. Amberger, S. Pirker, Progress in Computational Fluid Dynamics, an International Journal 12, 140 (2012)

[4] F. Blanc, F. Peters, E. Lemaire, Applied Rheology 21, 23735 (2011)

[5] J. Padding, A. Louis, Physical Review E 74, 031402 (2006)

[6] G. Gompper, T. Ihle, D. Kroll, R. Winkler, in Advanced computer simulation approaches for soft matter sciences III (Springer, 2009), pp. 1-87

[7] M. Hecht, J. Harting, T. Ihle, H.J. Herrmann, Physical Review E 72, 011408 (2005)

[8] C. Cierpka, R. Segura, R. Hain, C. Kähler, Measurement Science and Technology 21, 045401 (2010)

[9] P. Saffman, Journal of fluid mechanics 22, 385 (1965)

[10] P. Michael, Physics of Fluids (1958-1988) 8, 1263 (1965)

[11] W. Matson, M. Kalyankar, B.J. Ackerson, P. Tong, Physical Review E 71, 031401 (2005)

[12] E.S. Asmolov, Journal of Fluid Mechanics 381, 63 (1999)

[13] J.J. Stickel, R.L. Powell, Annu. Rev. Fluid Mech. 37, 129 (2005) 